\title{
The analysis of the performance of multi- beamforming in memory nonlinear power amplifier
}

\author{
Huiyong Li, Xun Li and Chen Wei
}

\begin{abstract}
With the increasingly diverse and complex requirements of radar systems and communication systems, the application of multifunction-phased array radar has become a trend, and the digital multi-beamforming technology plays a crucial role in it. In practice, power amplifier (PA) is an essential component in radar systems and communication systems. Unfortunately, it is always nonlinear to provide a high output power. With the purpose of a high output power and efficiency, it is necessary to study the influence of PA nonlinear characteristics on the digital multi-beamforming. In this paper, a form of the multi-beamforming signal and a nonlinear model with memory for PA are given. The output signal via the PA model has been analyzed subsequently. As the result of analysis, it can be found that the output signal is divided into the original signal and the interferential signal. The power ratio of original signal to interference signal can reflect the influence of PA nonlinear characteristics on the digital multi-beamforming. Finally, according to the ratio, the results of computer simulation show that the memory effect plays a key role for the small power signal, while the nonlinearity plays an important role for the large power signal.
\end{abstract}

\section{Introduction}

In recent years, with the development of the military radar technology and the increasing demand for information processing of communications, the application of integrated electronic information system with multifunction-phased array radar has become a trend, and the digital multibeamforming technology is crucial to the implementation of this system. At present, in contrast to the mature receiving digital multi-beamforming technology, the transmitted digital multi-beamforming technology is still under development. Transmitted multi-beamforming has many advantages. For example, when the radar array intends to search and track objects, the working mode of the common beam is time division, which means that only one job, tracking or searching, can be done at the same time. If the transmitted simultaneous multi-beamforming is used, the two work can be carried out at the same time by using two beams, which are added and synthesized in the digital side and can be transmitted simultaneously. However, because the transmitted signal is the sum of signals, the major

\footnotetext{
* Correspondence: hyli@uestc.edu.cn

School of Electronic Engineering, University of Electronic Science and Technology of China, Chengdu 611731, China
}

bottleneck of the realization of the transmitted simultaneous multi-beamforming is that the transmitted signal envelope is not constant. Therefore, it has a higher requirement on linearity of the transmitter power amplifier.

In order to design an optimal PA, it is necessary to analyze the effect of the PA nonlinearities on transmitted multi-beamforming firstly. The nonlinear distortion of PA has always been a hot research area. A memory PA model is proposed in [1] and the performance of behavioral models is analyzed in [2]. $\mathrm{H} \mathrm{Ku}$ has analyzed behavioral modeling of nonlinear RF power amplifiers with memory effects in [3]. The major consequence of memory effects has been introduced by W Bosch in [4]. The papers $[5,6]$ present that memory effects of PA in the wideband system are obvious. However, here the definition of wideband is the high ratio of the transmitted signal bandwidth to the device bandwidth, rather than the signal bandwidth to the carrier frequency. For a transmitted narrowband signal, the memory effects of the PA are evident when the signal bandwidth matches the device bandwidth [7]. The constant envelope signal in the nonlinear PA is studied in [8]. Kohls [9] has used a Bessel series fit to the measured amplifier transfer functions

\section{实}

(c) 2014 Li et al.; licensee Springer. This is an Open Access article distributed under the terms of the Creative Commons Attribution License (http://creativecommons.org/licenses/by/2.0), which permits unrestricted use, distribution, and reproduction in any medium, provided the original work is properly credited. 
with a computer model to predict third-order intermodulation product beam patterns. In [10], Hemmi describes the nonlinear PA response of an active linear array by a third-order polynomial and develops equations for the beam-pointing angle of the harmonics and intermodulation products. An active phased array multibeam antenna model, including the nonlinear Shimbo model of the amplifiers, has been developed and validated experimentally in [11], which is useful for solid state power amplifiers (SSPAs). In [12], only the AM-AM varieties are considered to affect the beamforming, regardless of the AM-PM varieties and memory effects.

In this paper, the principle of transmitted multibeamforming is presented in section 2 . Then, section 3.1 presents a model based on the Hammerstein model to describe the AM-AM varieties, AM-PM varieties and memory effects. The output signal model from PA is analyzed in section 3.2. The simulation results are shown in section 4. Finally, section 5 concludes the study. Assumed that each channel and array elements are ideal and each PA is identical. A narrowband signal is considered and the attention will be focused on the effects of the amplitude and phase nonlinearities.

\section{The principle of transmitting multi-beamforming}

As shown in Figure 1, consider an $L$-element array with elements uniformly spaced on the line of distance equal to $d$. The transmitted signals are $s_{1}(n), \ldots, s_{N}(n)$ and the weight vectors are $\mathbf{w}_{1}, \ldots, \mathbf{w}_{N}$.

$$
\begin{aligned}
& \mathbf{w}_{i}=\left[1, e^{j \varphi_{i}}, \cdots, e^{j(L-1) \varphi_{i}}\right]^{\mathrm{T}}, i=1, \cdots, N \\
& \varphi_{i}=\frac{2 \pi d \sin \theta_{i}}{\lambda}
\end{aligned}
$$

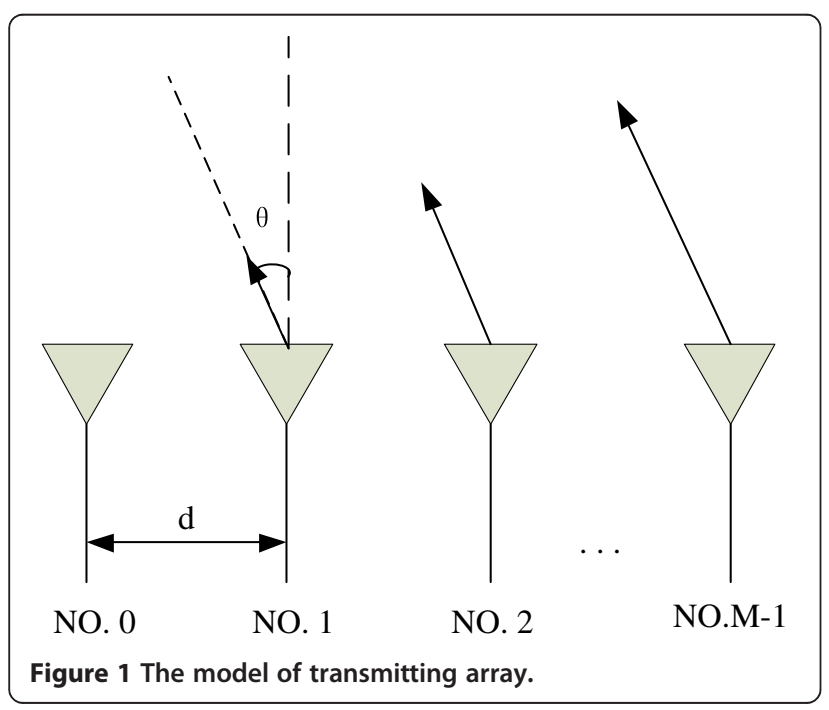

where $\varphi_{i}$ is the space phase of the $i$ th signal, $\lambda$ is the wavelength and $\theta_{i}$ is the transmitted angle of the $i$ th signal.

The space-power-function of transmitted multi-beam is given by

$$
f(\theta)=\left|\sum_{i=1}^{N} \mathbf{w}_{i}^{H} \mathbf{a}(\theta) s_{i}(n)\right|^{2}
$$

where $\boldsymbol{a}(\theta)=\left[1, e^{j \varphi}, \cdots, e^{j(L-1) \varphi}\right]^{T}$ is the steering vector of the scanning beam. When the transmitted signals are orthogonal and the powers of them are equal to each other, the multi-beam pattern can be written as

$$
E(\theta)=\frac{f(\theta)}{P_{s}}=\sum_{i=1}^{N}\left|\mathbf{w}_{i}^{H} \mathbf{a}(\theta)\right|^{2}
$$

where $P_{s}$ is the power of transmitted signal.

\section{The analysis of transmitted multi-beamforming based on nonlinear memory power amplifier}

\subsection{The model of PA}

A behavior model for PA can be divided into two types: the band-pass PA model for the RF signal processing and the baseband PA model for the envelope information processing. In practical engineering, the input signal of PA is a real signal with radio frequency. Therefore, the band-pass PA model can be very accurate to analyze a variety of components in the nonlinear devices, including harmonics, intermodulation, etc. However, in fact, the processed harmonics will not be transmitted. As the result, it is not convenient to analyze the signal of the band-pass PA model. As we all know, the useful information is carried only by the signal envelope. Moreover, nonlinear characteristics reflected by the behavioral model are only related to the signal amplitude, rather than the frequency. Therefore, a baseband PA model can be used for the signal analysis.

In this paper, the Hammerstein model (Figure 2), a modification of Volterra, has been used, which is given by:

$$
\begin{aligned}
& y(n)=\sum_{k=1}^{P} a_{k} x^{k}(n) \\
& z(n)=\sum_{i=0}^{M-1} h_{i} y(n-i)
\end{aligned}
$$

where $x(n), y(n)$, and $z(n)$ denote the input signal of the memoryless nonlinear PA model, memory linear PA and 


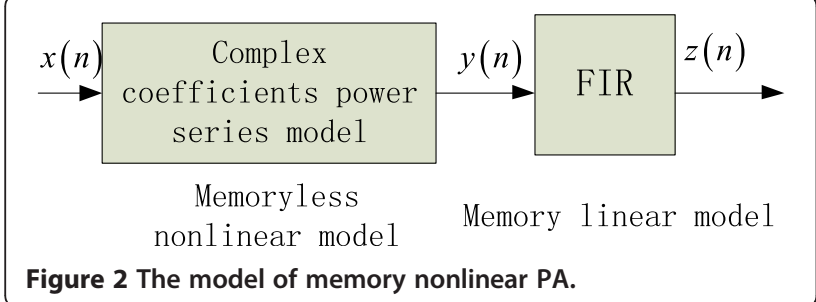

Hammerstein, respectively. $M$ is the memory depth and $P$ is the order of nonlinear system.

The memory linear model uses an $M$ - order FIR filter as description. In order to reflect its AM-AM and AM-PM characteristics simultaneously, the memoryless nonlinear model employs a complex coefficients power series model. Assume that $a_{k}=a_{I k}+j a_{Q k}, x(n)=r e^{j \theta}$. So,

$$
\begin{aligned}
y(n) & =\sum_{k=1}^{P} a_{k} x(n)|x(n)| k-1 \\
& =r e^{j \theta} \sum_{k=1}^{P} a_{k} r^{k-1} \\
& =\left[\sum_{k=1}^{P} a_{I l} r^{k}+j \sum_{k=1}^{P} a_{\mathrm{Q} k} r^{k}\right] e^{j \theta} \\
& =\sqrt{\left(\sum_{k=1}^{P} a_{I k} r^{k}\right)^{2}+\left(\sum_{k=1}^{P} a_{\mathrm{Q} k} r^{k}\right)^{2}} e^{j\left(\theta+\arctan \left(\sum_{k=1}^{P} a_{Q k} r^{k} / \sum_{k=1}^{P} a_{l k} r^{k}\right)\right)}
\end{aligned}
$$

The functions of AM-AM and AM-PM can be given by:

$$
\begin{aligned}
& A(r)=\sqrt{\left(\sum_{k=1}^{P} a_{I k} r^{k}\right)^{2}+\left(\sum_{k=1}^{P} a_{Q k} r^{k}\right)^{2}} \\
& \Phi(r)=\arctan \left(\sum_{k=1}^{P} a_{Q k} r^{k} / \sum_{k=1}^{P} a_{I k} r^{k}\right)
\end{aligned}
$$

From the above conclusion, power series with complex coefficients can describe the characteristics of AM-AM and AM-PM. If the PA has no memory, the output of the PA is determined only by the odd-component of the input signal. The even-component of the input signal will not affect the output of the PA, which can be expressed as:

$$
y(n)=\sum_{k=0}^{(P-1) / 2} a_{2 k+1}|x(n)|^{2 k} x(n)
$$

\subsection{The analysis of the transmitted multi-beam signal} based on the Taylor model

After frequency mixing of two transmitted real signals, the output signals can be represented as:

$$
\begin{aligned}
& s_{1}(t)=A_{1}(t) \cos \left(\omega_{0} t+\varphi_{1}(t)\right) \\
& s_{2}(t)=A_{2}(t) \cos \left(\omega_{0} t+\varphi_{2}(t)\right)
\end{aligned}
$$

The signal input into the nonlinear PA is given by:

$$
\begin{aligned}
s_{\text {in }}(t)= & A_{w_{1}} A_{1}(t) \cos \left(\omega_{0} t+\varphi_{1}(t)-\phi_{w 1}\right) \\
& +A_{w_{2}} A_{2}(t) \cos \left(\omega_{0} t+\varphi_{2}(t)-\phi_{w 2}\right)
\end{aligned}
$$

where $A_{w_{1}}, A_{w_{2}}$ and $\phi_{w_{1}}, \phi_{w_{2}}$ are the amplitudes and phases of the two weight vectors $w_{1}$ and $w_{2}$, respectively. $A_{1}(t), A_{2}(t)$ and $\varphi_{1}(t), \varphi_{2}(t)$ are the amplitudes and phases of the two signals, respectively. $\omega_{0}$ is the carrier frequency.

Even order harmonic component, produced by PA, can be removed by filters. Therefore, the odd-order harmonic component, which cannot be removed by filters, has a great effect on input signals. According to the power series model, after the signal passes the nonlinear PA, the output signal is given by:

$$
s_{\text {out }}(t)=a_{1} s_{\text {in }}(t)+a_{3} s_{\text {in }}(t)^{3}
$$

where $a_{1}, a_{3}$ is a real number.

Expanding the cubic term and removing the nonfundamental frequency component, we can get:

$$
\begin{aligned}
a_{3} s_{\text {in }}(t)^{3} \approx & a_{3}\left[\left(\frac{3}{4} A_{s_{1}}(t)^{3}+\frac{3}{2} A_{s_{1}}(t) A_{s_{2}}(t)^{2}\right) \cos \left(\omega_{0} t+\varphi_{1}(t)-\phi_{w_{1}}\right)\right. \\
& +\left(\frac{3}{4} A_{s_{2}}(t)^{3}+\frac{3}{2} A_{s_{2}}(t) A_{s_{1}}(t)^{2}\right) \cos \left(\omega_{0} t+\varphi_{2}(t)-\phi_{w_{2}}\right) \\
& +\left(\frac{3}{4} A_{s_{2}}(t) A_{s_{1}}(t)^{2}\right) \cos \left(w_{0} t+2\left(\varphi_{1}(t)-\phi_{w_{1}}\right)-\varphi_{2}(t)-\phi_{w_{2}}\right) \\
& \left.+\left(\frac{3}{4} A_{s_{1}}(t) A_{s_{2}}(t)^{2}\right) \cos \left(w_{0} t+2\left(\varphi_{2}(t)-\phi_{w_{2}}\right)-\varphi_{1}(t)-\phi_{w_{1}}\right)\right]
\end{aligned}
$$

where $A_{s_{1}}(t)=A_{w_{1}} A_{1}(t), A_{s_{2}}(t)=A_{w_{2}} A_{2}(t)$.

The real signal is a double-sideband signal. To analyze conveniently, we study one side of the spectrum, and the analytic signal of output is given by:

$$
\begin{aligned}
s_{\text {out }}(t) & =a_{1}\left(A_{s_{1}}(t) e^{j\left(\omega_{0} t+\varphi_{1}(t)-\phi_{w_{1}}\right)}+A_{s_{2}}(t) e^{j\left(\omega_{0} t+\varphi_{2}(t)-\phi_{w_{2}}\right)}\right) \\
& +a_{3}\left[\left(\frac{3}{4} A_{s_{1}}()^{3}+\frac{3}{2} A_{s_{1}}(t) A_{s_{2}}()^{2}\right) e^{j\left(\omega_{0} t+\varphi_{1}(t)-\phi_{w_{1}}\right)}\right. \\
& +\left(\frac{3}{4} A_{s_{2}}(t)^{3}+\frac{3}{2} A_{s_{2}}(t) A_{s_{1}}(t)^{2}\right) e^{j\left(\omega_{0} t+\varphi_{2}(t)-\phi_{w_{2}}\right)} \\
& +\left(\frac{3}{4} A_{s_{2}}(t) A_{s_{1}}(t)^{2}\right) e^{j\left(w_{0} t+2\left(\varphi_{1}(t)-\phi_{w_{1}}\right)-\varphi_{2}(t)-\phi_{w_{2}}\right)} \\
& \left.+\left(\frac{3}{4} A_{s_{1}}(t) A_{s_{2}}(t)^{2}\right) e^{j\left(w_{0} t+2\left(\varphi_{2}(t)-\phi_{w_{2}}\right)-\varphi_{1}(t)-\phi_{w_{1}}\right)}\right]
\end{aligned}
$$


According to expression in (12), whether the nonlinear term is the same as the original signal is related to the input signal. Under amplitude modulation, the desired signal can be represented as:

$$
s_{\text {desire }}(t)=a_{1}\left(A_{s_{1}}(t) e^{j\left(\omega_{0} t+\varphi_{1}(t)-\phi_{w_{1}}\right)}+A_{s_{2}}(t) e^{j\left(\omega_{0} t+\varphi_{2}(t)-\phi_{w_{2}}\right)}\right)
$$

All items of $a_{3}$ in expression (12), which are different from previous signals, are interference signals.

$$
\begin{aligned}
s_{\text {jam }}(t)= & a_{3}\left[\left(\frac{3}{4} A_{s_{1}}(t)^{3}+\frac{3}{2} A_{s_{1}}(t) A_{s_{2}}(t)^{2}\right) e^{j\left(\omega_{0} t+\varphi_{1}(t)-\phi_{w_{1}}\right)}\right. \\
& +\left(\frac{3}{4} A_{s_{2}}(t)^{3}+\frac{3}{2} A_{s_{2}}(t) A_{s_{1}}(t)^{2}\right) e^{j\left(\omega_{0} t+\varphi_{2}(t)-\phi_{w_{2}}\right)} \\
& +\left(\frac{3}{4} A_{s_{2}}(t) A_{s_{1}}(t)^{2}\right) e^{j\left(\omega_{0} t+2\left(\varphi_{1}(t)-\phi_{w_{1}}\right)-\phi_{2}(t)-\phi_{w_{2}}\right)} \\
& \left.+\left(\frac{3}{4} A_{s_{1}}(t) A_{s_{2}}(t)^{2}\right) e^{j\left(\omega_{0} t+2\left(\varphi_{2}(t)-\phi_{w_{2}}\right)-\varphi_{1}(t)-\phi_{w_{1}}\right)}\right]
\end{aligned}
$$

When it is not an amplitude modulation, the cubic term contains a part of desired signal component, and the desired signal can be represented as:

$$
\begin{aligned}
s_{\text {desire }}(t) & =\left[a_{1} A_{s_{1}}+a_{3}\left(\frac{3}{4} A_{s_{1}}{ }^{3}+\frac{3}{2} A_{s_{1}} A_{s_{2}}{ }^{2}\right)\right] e^{j\left(\omega_{0} t+\varphi_{1}(t)-\phi_{w_{1}}\right)} \\
& +\left[a_{1} A_{s_{2}}+a_{3}\left(\frac{3}{4} A_{s_{2}}{ }^{3}+\frac{3}{2} A_{s_{2}} A_{s_{1}}{ }^{2}\right)\right] e^{j\left(\omega_{0} t+\varphi_{2}(t)-\phi_{w_{2}}\right)}
\end{aligned}
$$

The interference signal can be represented as:

$$
\begin{aligned}
s_{\mathrm{jam}}(t) & =a_{3}\left[\left(\frac{3}{4} A_{s_{2}} A_{s_{1}}{ }^{2}\right) e^{j\left(\omega_{0} t+2\left(\varphi_{1}(t)-\phi_{w_{1}}\right)-\varphi_{2}(t)-\phi_{w_{2}}\right)}\right. \\
& \left.+\left(\frac{3}{4} A_{s_{1}} A_{s_{2}}{ }^{2}\right) e^{j\left(\omega_{0} t+2\left(\varphi_{2}(t)-\phi_{w_{2}}\right)-\varphi_{1}(t)-\phi_{w_{1}}\right)}\right]
\end{aligned}
$$

According to expression in (14) and (16), on the condition of amplitude modulation, the power of interference signal mainly consists of the signal which has the same weight vector with the desired signal, and the power of interference signal has the same pointing direction with the desired signal. However, on the condition of nonamplitude modulation, it is the weight vectors $w_{1}^{\mathrm{H} 2} w_{2}$ and $w_{2}^{\mathrm{H} 2} w_{1}$ that determining the pointing direction of interference signal power.

\subsection{The analysis of the transmitted multi-beam signal based on the Hammerstein model}

A complex envelope of the input signal which is the sum of two narrow-band signals can be expressed by:

$$
\begin{aligned}
x(n) & =A_{w_{1}} A_{1}(n) e^{j \varphi_{1}(n)-\phi_{w_{1}}}+A_{w_{2}} A_{2}(n) e^{j \varphi_{2}(n)-\phi_{w_{2}}} \\
& =r_{1}(n) e^{j \varphi_{1}(n)-\phi_{w_{1}}}+r_{2}(n) e^{j \varphi_{2}(n)-\phi_{w_{2}}}
\end{aligned}
$$

where $A_{w_{1}}, A_{w_{2}}, \phi_{w_{1}}$ and $\phi_{w_{2}}$ are the amplitudes and phases of the two weight vectors, and $A_{1}(n), A_{2}(n), \varphi_{1}(n)$, and $\varphi_{2}(n)$ are the amplitudes and phases of the two signals. $r_{1}(n)=A_{w_{1}} A_{1}(n)$ and $r_{2}(n)=A_{w_{2}} A_{2}(n)$.

After the signal passes the memoryless nonlinear model, the output signal is given by

$$
\begin{aligned}
y(n) & =a_{1} x(n)+a_{3} x(n)|x(n)|^{2} \\
& =\left(a_{1}+a_{3} r^{2}(n)\right) x(n)
\end{aligned}
$$

where $r^{2}(n)=r_{1}(n)^{2}+r_{2}(n)^{2}+2 r_{1}(n) r_{2}(n) \cos \left[\varphi_{1}(n)-\right.$ $\left.\phi_{w_{1}}-\varphi_{2}(n)+\phi_{w_{2}}\right]$.

If the input signals have constant envelopes, that is $r_{1}(n)=r_{1}$ and $r_{2}(n)=r_{2}$ then:

$$
\begin{aligned}
y(n)= & {\left[a_{1} r_{1}+a_{3} r_{1}\left(r_{1}^{2}+r_{2}^{2}\right)\right] e^{j \varphi_{1}(n)-\phi_{w_{1}}} } \\
& +2 a_{3} r_{1}^{2} r_{2} \cos \left[\varphi_{1}(n)-\phi_{w_{1}}-\varphi_{2}(n)+\phi_{w_{2}}\right] e^{j \varphi_{1}(n)-\phi_{w_{1}}} \\
& +\left[a_{1} r_{2}+a_{3} r_{2}\left(r_{1}^{2}+r_{2}^{2}\right)\right] e^{j \varphi_{2}(n)-\phi_{w_{2}}} \\
& +2 a_{3} r_{2}^{2} r_{1} \cos \left[\varphi_{1}(n)-\phi_{w_{1}}-\varphi_{2}(n)+\phi_{w_{2}}\right] e^{j \varphi_{2}(n)-\phi_{w_{2}}}
\end{aligned}
$$

We notice that the first and third coefficients are time-independent complexes, so they can be regarded as original signals. However, the second and fourth coefficients are time-dependent complexes, which means that the information carried by the signal envelope has changed. Hence, they should be considered as interferential signals. The effect of nonlinearity on the transmitted signal can be measured by the power ratio of the original signal to interference.

The output of memoryless PA model is seen as the input of linear FIR filter. Assume that the memory depth is 2. Then, the output signal of memory PA model can be represented as:

$$
z(n)=h_{0} y(n)+h_{1} y(n-1)
$$

According to the concept of phase modulation and frequency modulation, the phase difference of $\varphi(n-1)$ and $\varphi(n)$ is decided by the modulation signal. So, $\varphi(n-1)$ 
and $\varphi(n)$ are different information. Finally, Equation 10 also can be described as:

$$
\begin{aligned}
z(n)= & h_{0}\left[a_{1} r_{1}+a_{3} r_{1}\left(r_{1}^{2}+r_{2}^{2}\right)\right] e^{j \varphi_{1}(n)-\phi_{w_{1}}} \\
& +h_{0}\left[a_{1} r_{2}+a_{3} r_{2}\left(r_{1}^{2}+r_{2}^{2}\right)\right] e^{j \varphi_{2}(n)-\phi_{w_{2}}} \\
& +h_{1}\left[a_{1} r_{1}+a_{3} r_{1}\left(r_{1}^{2}+r_{2}^{2}\right)\right] e^{j \varphi_{1}(n-1)-\phi_{w_{1}}} \\
& +h_{1}\left[a_{1} r_{2}+a_{3} r_{2}\left(r_{1}^{2}+r_{2}^{2}\right)\right] e^{j \varphi_{2}(n-1)-\phi_{w_{2}}} \\
& +2 h_{0} a_{3} r_{1}^{2} r_{2} \cos \left[\varphi_{1}(n)-\phi_{w_{1}}-\varphi_{2}(n)+\phi_{w_{2}}\right] e^{j \varphi_{\varphi 1}(n)-\phi_{w_{1}}} \\
& +2 h_{1} a_{3} r_{1}^{2} r_{2} \cos \left[\varphi_{1}(n-1)-\phi_{w_{1}}-\varphi_{2}(n-1)+\phi_{w_{2}}\right] e^{j \varphi_{1}(n-1)-\phi_{w_{2}}} \\
& +2 h_{0} a_{3} r_{2}^{2} r_{1} \cos \left[\varphi_{1}(n)-\phi_{w_{1}}-\varphi_{2}(n)+\phi_{w_{2}}\right] e^{j \varphi_{2}(n)-\phi_{w_{2}}} \\
& +2 h_{1} a_{3} r_{2}^{2} r_{1} \cos \left[\varphi_{1}(n-1)-\phi_{w_{1}}-\varphi_{2}(n-1)+\phi_{w_{2}}\right] e^{j \varphi_{2}(n-1)-\phi_{w_{2}}}
\end{aligned}
$$

According to the expression in (21), it can be seen that the first and second signals are still original signals. However, the third and fourth signals have different phases from the original signals, and the rest of the signals have become new signals which the amplitudes vary with time. That is to say, except for the first and second signals, the others are interferential signals.

\section{Simulation}

Consider a 16-element array with elements uniformly spaced on the line of distance equal to half wavelength. Two transmitted beams are assumed to be transmitted at angle $30^{\circ}$ and $-20^{\circ}$. Several null points are set at angle $-10^{\circ}$, $10^{\circ}$, and $50^{\circ}$. One thousand sampling points are chosen. The transmitted signals are two linear frequency modulation signals with $s_{1}(t)=A_{s_{1}} e^{j K t^{2}}+n_{1}(t)$ and $s_{2}=A_{s_{2}} e^{j 2 \pi B t+j K t^{2}}+n_{2}(t)$, where $K=B / T . \quad B=10 \mathrm{M}$ is the bandwidth of the signal and $T$ is pulse period, $T=$
$N T_{s}=N / f_{s}, N=1,000$ is the sampling numbers and $f_{s}=$ $3.01 / 2 \times 10^{9} \mathrm{~Hz}$ is the sampling frequency. $n_{1}(t)$ and $n_{2}$ $(t)$ are system thermal noises. The transmitted signal before it is input to PA has an $\mathrm{SNR}=90 \mathrm{~dB}$. Although the amplitudes, $A_{s_{1}}$ and $A_{s_{2}}$, are the same, the two signals which have different carrier frequencies are orthogonal. The first coefficient of PA is $a_{1}=1$. The first tap coefficient of FIR is $h_{0}=1$ and the memory depth is 2 . The beam pattern affected by the nonlinear PA is produced by a beamforming technique based on orthogonal projection algorithm.

$a_{1} / a_{3}$ is set to measure the memoryless nonlinearity of PA and $h_{0} / h_{1}$ is used to measure the memory effect of PA. The effect of nonlinearity PA on the multibeamforming at $-20^{\circ}$ can be shown by changing the two parameters.

Figure 3 and Figure 4 show AM-AM and AMPM variation curves versus different PA coefficients. According to these figures, it can be found that the linearity of $a_{3}=-0.01 \times(2+j)$ is less than $a_{3}=-0.01 \times(1+j)$.

The power ratio of the original signal to the interference can show the effect of the nonlinearity of PA on the input signal. Figure 5 shows that when $h_{1}=10^{-2}$ and the input signal power is below $7 \mathrm{dBm}$, the power ratio keeps $40 \mathrm{~dB}$ with different nonlinearities. It can be explained that when the input signal power is small, the memory effect plays a dominant role. On the other hand, when the input signal power is $20 \mathrm{dBm}$, owing to the nonlinearity, the ratio of $a_{3}=-0.01 \times(2+j)$ is more than $a_{3}=-0.01 \times(1+j)$.

Equation 9 shows that the equation value is significant only if $a_{3}$ and $h_{1} a_{1}$ have the same dimension, which means

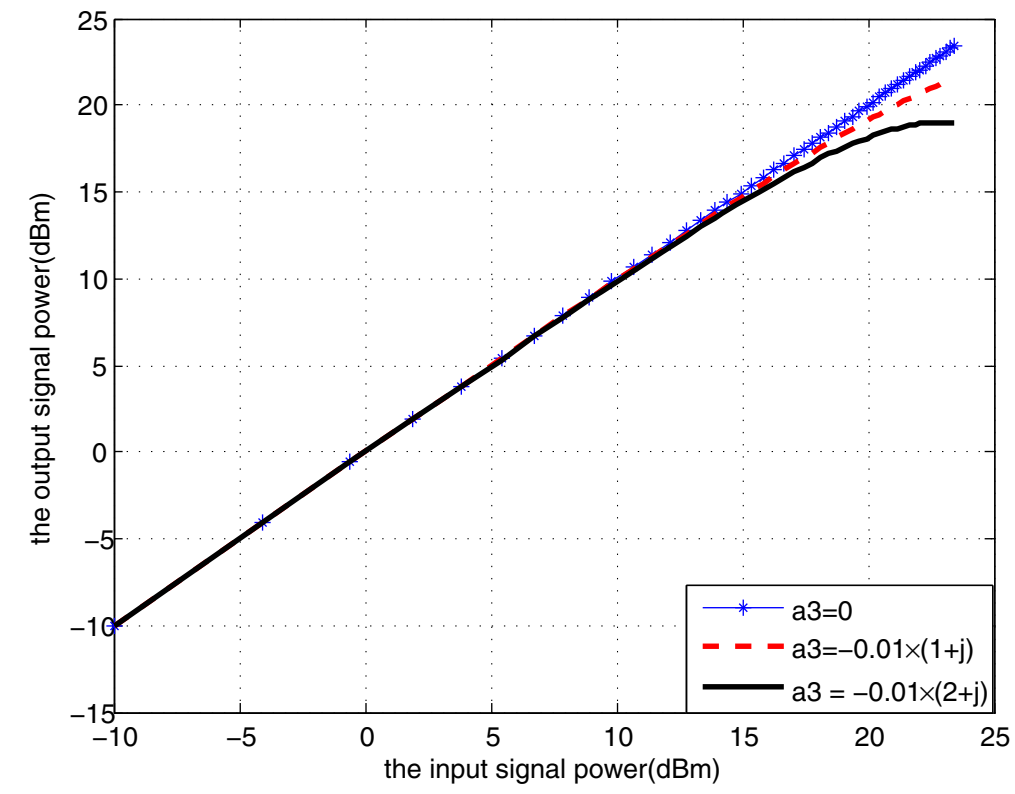

Figure 3 AM-AM conversion. 


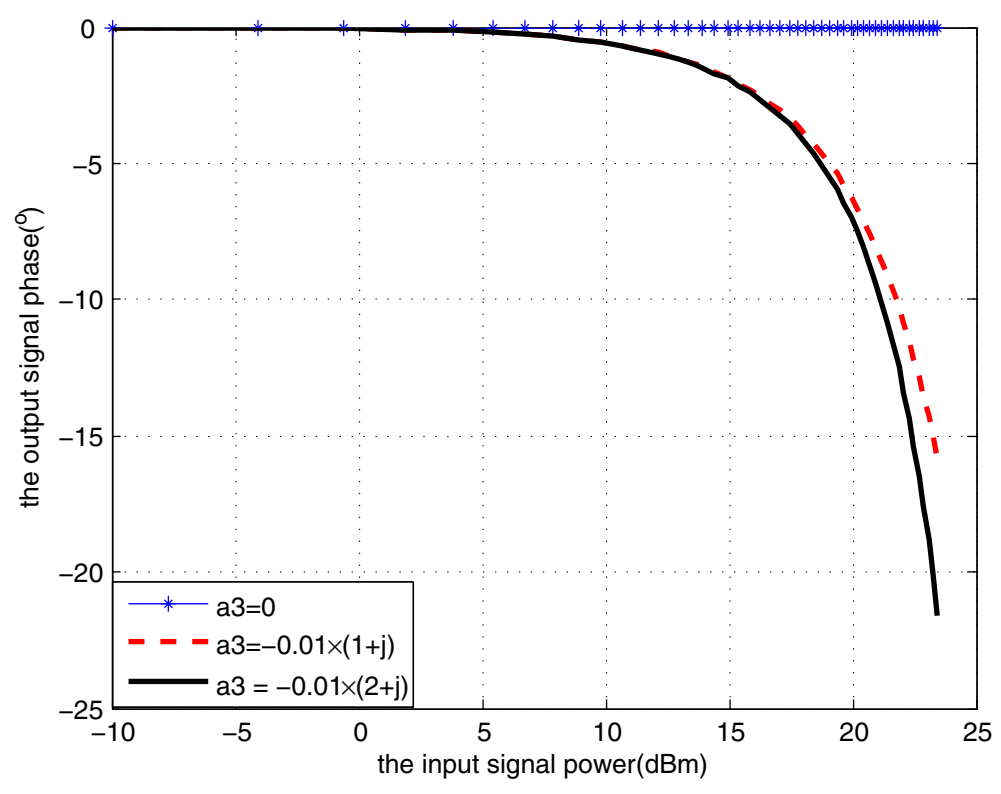

Figure 4 AM-PM conversion.

we can ignore the effect of $h_{1} a_{3}$ on beamforming. In addition, the nonlinearity represents gain compression characteristics. So, $a_{3}$ is negative and $h_{1}$ is positive. Under this premise, the total power of interference can be considered as a positive, when the memory effect plays a major role. The power of interference becomes weak as the memory effect is reduced. On the contrary, when nonlinearity plays a key role, the total power of interference is a negative.
The power of interference increases as the memory effect is reduced. This is why the power ratio has an upward trend.

To measure the memoryless nonlinearity of PA, we assumed that $a_{3}=-0.01 \times(x+j), h_{1}=10^{-5}$ and $A_{1}=19.3$ $\mathrm{dBm}$, where $x$ is an independent variable. Figure 6 shows that when the input signal power $A_{1}$ is $19.3 \mathrm{dBm}$, the power ratio of the different $h_{1}$ is almost identical. However, when $A_{1}$ is $9.8 \mathrm{dBm}$, the power ratio of the

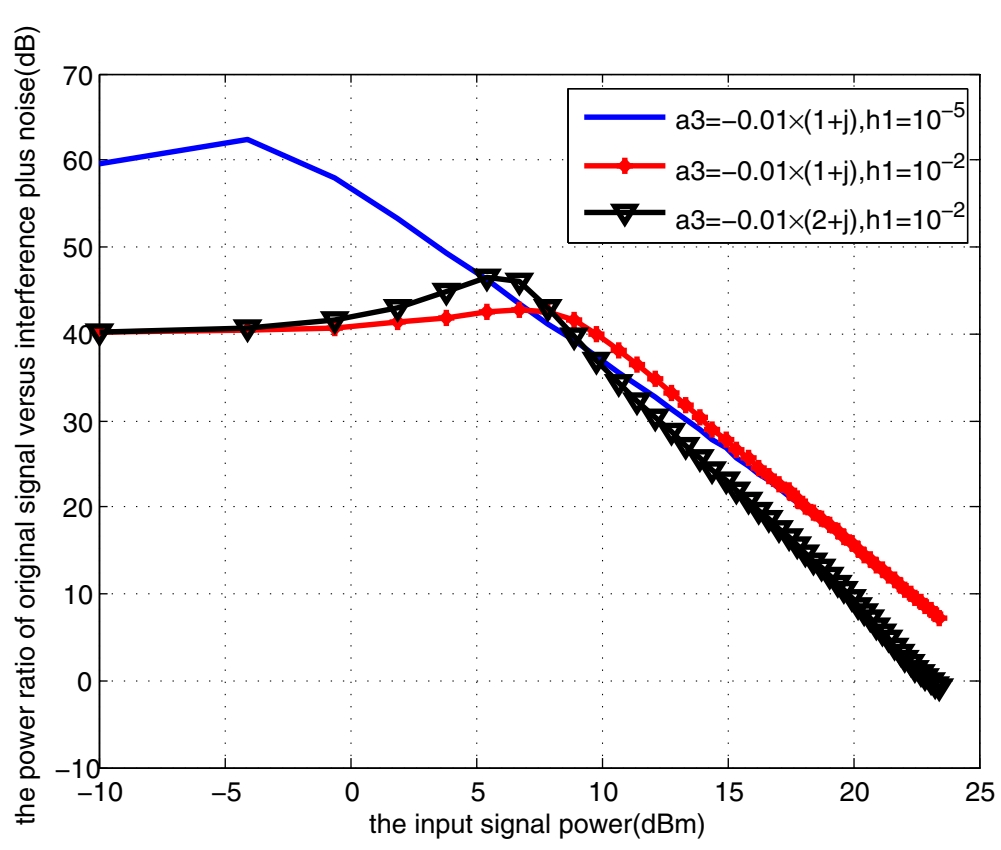

Figure 5 The power ratio of original signal versus interference plus noise of input signal. 


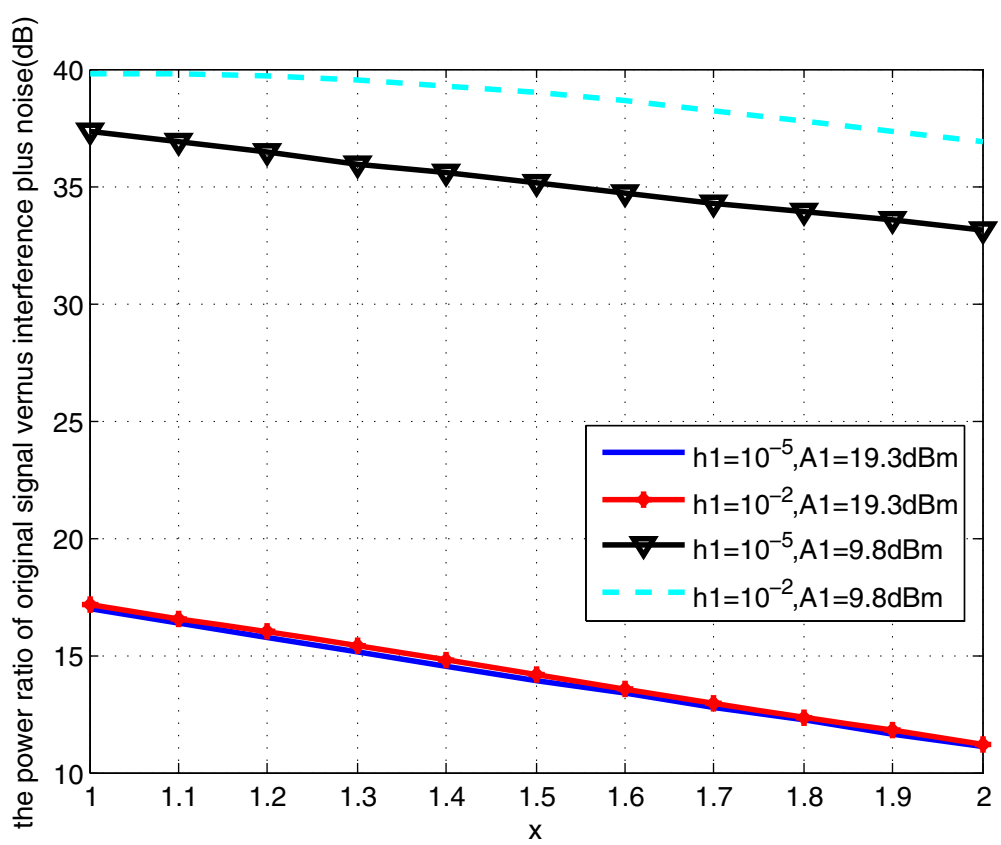

Figure 6 The power ratio of original signal versus interference plus noise with $x$.

different $h_{1}$ is different. The same conclusion can be found in Figure 6.

$a_{3}=-0.01 \times(1+j), A_{1}=9.8 \mathrm{dBm}$, and $A_{1}=19.3 \mathrm{dBm}$ are assumed to measure the memory effect of PA. From Figure 7, it can be seen that when the input signal power $A_{1}$ is $9.8 \mathrm{dBm}, h_{1}$ which is more than $10^{-4}$ will affect the ratio. However, it hardly affects the ratio when $h_{1}$ is less than $10^{-5}$. On the other hand, when the power of input signal
$A_{1}$ is $19.3 \mathrm{dBm}$, it is a turning point that $h_{1}$ is $10^{-2}$. So, it is concluded that the low power of input signal is sensitive to the memory effect. The reason for the upward trend in Figure 7 has already been explained in Figure 5 .

\section{Conclusion}

In this paper, starting from the signal form, the output signal of PA is obtained via the establishment of the PA

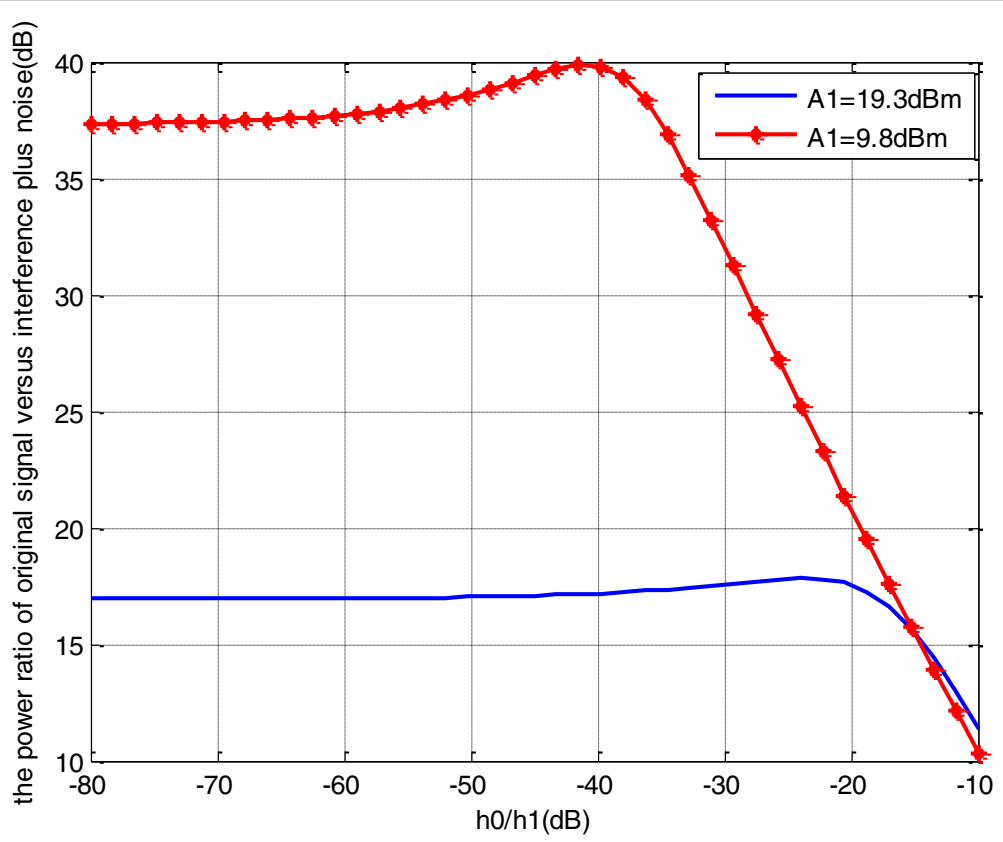

Figure 7 The power ratio of original signal versus interference plus noise with memory effect. 
model. Then the influence of the memory effect and nonlinearity of PA is presented by analyzing the composition of the final output signal. Lastly, computer simulations verify that the memory effect plays a key role, in the presence of the small power signal, while the nonlinearity plays an important role in the presence of the large power signal. A detailed theoretical basis and reference to linearize PA is provided in this paper for other researchers. When the transmitted signals are small power signals, the memory effect should gain attention in linearizing PA. However, if large power signals are transmitted, the importance of the nonlinearity of PA should be attached.

\section{Competing interests}

The authors declare that they have no competing interests.

\section{Acknowledgements}

This research was supported by Applied Basic Research Programs of Sichuan Province (No. 2013JY0004), National Natural Science Foundation of China (No. 61371184) and the Fundamental Research Funds for the Central Universities (No. ZYGX2012J018).

Received: 16 January 2014 Accepted: 24 March 2014

Published: 16 April 2014

\section{References}

1. L Ding, GT Zhou, DR Morgan, Z Ma, JS Kenney, J Kim, CR Giardina, A robust digital baseband predistorter constructed using memory polynomials. IEEE Trans. Commun. 52(1), 159-165 (2004)

2. M Isaksson, D Wisell, D Ronnow, A comparative analysis of behavioral models for RF power amplifiers. IEEE Trans. Microw. Theory Tech. 54(1), 348-359 (2006)

3. H Ku, JS Kenney, Behavioral modeling of nonlinear RF power amplifiers considering memory effects. IEEE Trans. Microw. Theory Tech. 51(12), 2495-2504 (2003)

4. W Bosch, G Gatti, Measurement and simulation of memory effects in predistortion linearizers. IEEE Trans. Microw. Theory Tech. 37, 1885-1890 (1989)

5. JHK Vuolevi, T Rahkonen, JPA Manninen, Measurement technique for characterizing memory effects in RF power amplifiers. IEEE Trans. Microw. Theory Tech 49, 1383-1388 (2001)

6. J Kim, K Konstantinou, Digital predistortion of wideband signals based on power amplifier model with memory. Electron. Lett. 37(23), 1417-1418 (2001)

7. H Ku, M Mckinley, JS Kenney, Quantifying memory effects in RF power amplifiers. IEEE Trans. Microw. Theory Tech. 50(12), 2843-2849 (2002)

8. $Y W u, Y L i u$, The analysis of the effect of high power amplifier and bandwidth to the constant envelop guidance signal. J Telemetry. Tracking. Command. 32(3), 14-20 (2011)

9. EC Kohls, EP Ekelman, Al Zaghloul, FT Assal, Intermodulation and bit-error ratio performance of a Ku-band multibeam high-power phased array, in Proceedings of the IEEE International Symposium on Antennas and Propagation, vol. 3 (IEEE, Piscataway, 1995), pp. 1404-1408

10. C Hemmi, Pattern characteristics of harmonic and intermodulation products in broadband active transmit arrays. IEEE Trans. Antennas Propag. 50(6), 858-865 (2002)

11. KJ Maalouf, E Lier, Theoretical and experimental study of interference in multibeam active phased array transmit antenna for satellite communications. IEEE Trans. Antennas Propag. 52(2), 587-592 (2004)

12. EC Real, DP Charette, Non-linear amplifier effects in transmit beamforming arrays, in Proceedings of the IEEE International Conference on Acoustics Speech Signal Processing, vol. 5 (IEEE, Piscataway, 1995), pp. 3635-3638

doi:10.1186/1687-6180-2014-52

Cite this article as: Li et al:: The analysis of the performance of multibeamforming in memory nonlinear power amplifier. EURASIP Journal on Advances in Signal Processing 2014 2014:52.

\section{Submit your manuscript to a SpringerOpen ${ }^{\circ}$ journal and benefit from:}

- Convenient online submission

- Rigorous peer review

- Immediate publication on acceptance

- Open access: articles freely available online

- High visibility within the field

- Retaining the copyright to your article

Submit your next manuscript at $\gg$ springeropen.com 